\title{
LA IMAGEN DE PLATÓN EN LA CRÍTICA DE LA RAZÓN PURA*
}

\section{THE PICTURE OF PLATO IN THE CRITIQUE OF PURE REASON}

\author{
Danilo BASTA** \\ Universidad de Belgrado
}

\begin{abstract}
RESUMEN: Este artículo parte de la tesis planteada por Paul Natorp en su famoso libro La doctrina de las ideas de Platón (1903), según la cual la filosofía de Platón incluye muchas cualidades que anticipan a Kant mientras el pensamiento de Kant es una vuelta a Platón. En efecto, no se puede poner en duda el hecho de que en el pensamiento de Platón existen algunas características que por su significado anticipan el esfuerzo de Kant de examinar críticamente las facultades del entendimiento humano, mientras algunos componentes de la actitud crítica de Kant aluden a la filosofía de Platón. Sin embargo, Platón no fue un Kant en potencia ni Kant un Platón actualizado. Incluso aunque Kant era completamente consciente de que las ideas (Formas) de Platón poseen ante todo una dimensión ontológico-especulativa, de acuerdo con sus propios intereses filosóficos las orientó hermenéuticamente hacia la ética y la política, abriendo así una nueva posibilidad de comprender la esencia misma de la filosofía de Platón. Con su imagen de Platón, Kant mostró al mismo tiempo algunos de sus propios rasgos de filósofo crítico.
\end{abstract}

Palabras clave: Teoría de las Ideas, filosofía práctica, crítica, filosofía política, virtud, dignidad de la filosofía.

* Título original: «Das Plato-Bild in der Kritik der reinen Vernunft», traducido al castellano por Óscar Cubo Ugarte.

** Catedrático de Filosofía del Derecho. 
ABSTRACT: The point of departure in this article is the claim raised by Paul Natorp in his famous book Plato's Doctrine of Ideas (1903) that Plato's philosophy includes many features that anticipate Kant while Kant's thought points back to Plato. Indeed, one cannot doubt the fact that there are some characteristics in Plato's thinking which by their intention and foresight point to Kant's endeavour to critically examine the powers of the human mind, while some components of Kant's critical stance refer to Plato's philosophy. Nevertheless, Plato is not just a Kant in potentia, nor is Kant only an accomplished Plato. Even though Kant was well aware that ideas (forms) in Plato possess primarily an ontological function speculatively conceived, Kant, in keeping with his own philosophical strivings, hermeneutically shifted their import to ethics and politics, thus opening a new possibility of productively understanding the very core of Plato's philosophy. With his Plato picture, Kant simultaneously draws some essential traits of his own portrait as a critical philosopher.

KeYwords: Doctrine of Ideas, Practical Philosophy, Criticism, Political Philosophy, Virtue, Dignity of Philosophy.

Uno de los máximos representantes del neokantismo de la escuela de Marburgo, Paul Natorp, publicó hace un siglo (1903) su famoso libro La Doctrina de las Ideas de Platón ${ }^{1}$ que, sin duda, supuso un renovado y muy importante impulso a la investigación de la filosofía de Platón. Dieciocho años después (1921) apareció la segunda edición del mencionado libro; esta vez el autor consideró indispensable añadir un apéndice metacrítico al libro que había escrito en 1920 con el título de «Logos-Psyche-Eros». En un pasaje del mencionado apéndice, que es muy amplio e imprescindible para la comprensión del libro, Natorp rechazaba decididamente aquella objeción dirigida contra él de querer interpretar (o, mejor dicho, distorsionar) a Platón como si se tratara de un kantiano anterior a Kant o más bien de un kantiano de Marburgo previo a Marburgo. Pero a continuación añadía lo siguiente: «aquel que no percibe en Platón ciertos rasgos de Kant, y viceversa, es que ha debido entender a los dos bastante mal $»^{2}$.

\footnotetext{
${ }^{1}$ Paul Natorp, P. Platos Ideenlehre. Hamburg: Felix Meiner (1994).

${ }^{2}$ Ibid., p. 462.
} 
Ciertamente, el creativo fundador del idealismo filosófico y el creador del idealismo crítico tienen numerosos puntos de unión, a menudo visibles y a veces también invisibles. Es indiscutible que en el pensamiento de Platón -y especialmente en el centro de su pensamiento, esto es, en su doctrina de las ideasse contienen motivos en los que se advierte y anticipa la empresa kantiana de examinar críticamente la razón humana, por lo que respecta a su capacidad y sus límites. Y, por otro lado, algunos elementos del criticismo kantiano (por resumir en una palabra su entero esfuerzo y sus objetivos) también repercuten a su vez en la filosofía de Platón. En este sentido, no cabe sino estar de acuerdo con la opinión de Natorp. Ahora bien, con el fin de impedir posibles malentendidos, conviene también señalar que ni Platón es un kantiano en potencia ni Kant un Platón acabado y perfeccionado. En el caso de que la relación recíproca entre ambos pensadores fuera interpretada de este modo, se produciría un daño hermenéutico tanto al uno como al otro, y la consecuencia de todo ello no sería más que una representación unilateral y simplificada de estos dos gigantes de la filosofía idealista ${ }^{3}$.

De los dos pensadores griegos más importantes: Platón y Aristóteles, Kant se sentía mucho más cerca de Platón y lo prefería antes que al segundo. Aunque en sus obras mencione de una manera bastante parecida a ambos autores, lo cual constituye evidentemente sólo una indicación formal, Platón ocupa una posición más elevada que Aristóteles a los ojos de Kant. Si leemos por ejemplo la última parte de la Crítica de la razón pura, o sea, el cuarto capítulo de la «Doctrina trascendental del método», que lleva por título «La historia de la razón pura», que sólo está expuesta en forma de indicaciones, entonces podremos comprobar sin dificultad que Kant caracteriza a Aristóteles como el líder de los empiristas y a Platón como el líder de los noologistas. Además, Platón es caracteri-

3 De una manera amplia, abarcadora y sistemática, siguiendo algunas ideas, opiniones y estímulos que había encontrado en algunos neokantianos, sobre todo en Hermann Cohen, Ottomar Wichmann investigó y discutió en su estudio comparativo: Platon und Kant (Weidmannische Buchhandlung, Berlin, 1920) la relación entre Platón y Kant y sus más hondas aspiraciones filosóficas. En el centro de su investigación colocó la cuestión de la certeza y de la incondicionalidad (véanse por ejemplo las páginas 38, 46, 199-200) donde él expone las semejanzas y las diferencias entre Platón y Kant. Conviene también mencionar a este respecto que, en el marco de sus investigaciones, Wichmann muestra en algunos lugares, especialmente en las cuestiones relativas a la filosofía moral, una inclinación abierta por Platón sin reducir con ello, empero, el inmenso significado y el enorme mérito de la filosofía trascendental de Kant. 
zado en este mismo lugar de una manera halagadora como «el filósofo más destacado de lo intelectual $»^{4}$. En otras palabras, mientras que Aristóteles es el creador de aquella orientación filosófica que en la modernidad continuó Locke, Platón es el fundador de aquella corriente que Leibniz amplía y profundiza en la modernidad. Según esto, el «bosquejo superficial» de Kant sobre las modificaciones (revoluciones) más significativas llevadas a cabo en el desarrollo de la metafísica otorga, de un modo ciertamente discreto pero suficientemente claro, el predominio a Platón en relación con Aristóteles, un predominio que no puede ser menoscabado en modo alguno cuando Kant caracteriza de pasada el sistema de Platón como «místico». Todo esto no puede ser en absoluto una casualidad. Más bien está estrechamente ligado con los esfuerzos predominantes y el carácter fundamental de la filosofía trascendental kantiana y su crítica de la razón.

La fuente más completa e importante para la imagen de Platón esbozada por Kant, no sólo dentro de la Crítica de la razón pura sino también en toda su obra, se encuentra en el primer apartado del primer libro de la «Dialéctica trascendental», donde Kant discute el tema de las ideas. Estas pocas páginas merecen nuestra mayor atención, no sólo porque en ese lugar, en comparación con sus otras obras, es donde Kant más se detiene en relación con Platón, sino asimismo porque en esta ocasión examina, también en cuanto al contenido, todo lo que es substancial y, en esa medida, decisivo para su imagen de Platón. No hay ningún otro sitio ni ninguna otra obra donde Kant hable sobre Platón con tanta comprensión y tanto reconocimiento, con tan fina simpatía y compenetración con el pensamiento de ese "filósofo sublime» (como Kant mismo lo caracteriza), y de una manera tan adecuadamente ponderada. En esto tiene una importancia esencial que, justamente en su confrontación con la filosofía de Platón y su punto nuclear, la doctrina de las ideas, Kant formule y a la vez aplique un famoso principio hermenéutico que, posteriormente, se haría célebre gracias a Schleiermacher (por lo cual se le hace a Kant, como verdadero creador de este principio, una grande y manifiesta injusticia). Tras haber señalado que Platón entiende con la expresión de «ideas» algo que no sólo es extraído de los sentidos, sino que sobrepasa con mucho los mismos conceptos del entendimiento, de los que se ocupó Aristóteles (con lo que ya expresa la preferencia de Platón sobre Aristóteles), Kant

\footnotetext{
${ }^{4}$ Immanuel Kant. Crítica de la razón pura, B 881.
} 
añade lo siguiente: «me limito a observar que no es raro que, comparando los pensamientos expresados de un autor acerca de su tema, tanto en el lenguaje ordinario como en los libros, lleguemos a entenderle mejor de lo que él se ha entendido a sí mismo, en la medida en que al no precisar suficientemente su concepto, dicho autor hablaba o pensaba incluso de forma contraria a lo que era su propio objetivo"s.

Todo lo que Kant escribe a partir de ese momento hay que leerlo como una comprensión mejor de Platón que lo que él mismo se había comprendido. La más significativa idea en relación con esta mejor comprensión de Platón, la idea que constituye el hilo conductor fiable, dice así: «Platón halló sus ideas preferentemente en todo lo práctico, es decir, en aquello que se basa en la libertad, la cual, a su vez, está bajo conocimientos que son un producto genuino de la razón» ${ }^{6}$.

Por medio de ello Kant establece claramente la perspectiva hermenéutica bajo la que él había visto la doctrina de las ideas de Platón. Con independencia de que se pueda observar (o incluso objetar) que esta perspectiva es demasiado kantiana, es decir, que la doctrina de las ideas de Platón es expuesta por Kant desde su propio posicionamiento filosófico y desde el punto de vista (y desde el primado) de la razón pura práctica, es indiscutible que Kant por medio de su comprensión de Platón abre posibilidades muy fructíferas a la hora de entender el núcleo central de la filosofía platónica, pues a través de esta interpretación Kant desplaza el peso de la discusión sobre la doctrina de las ideas de Platón del terreno del conocimiento especulativo al terreno de la filosofía práctica, esto es, al terreno de aquello que «se basa en la libertad». En otras palabras, el propósito de Kant era formar una imagen de Platón y de su doctrina de las ideas, acentuando y desplegando el sentido profundamente práctico de las mismas ${ }^{7}$. Ciertamente, él era consciente de que las ideas en Platón tienen una clara dimensión especulativo-ontológica; pero él orientaba sus intereses hermenéuticos fundamentales a su significación ético-política. El motivo de ello lo encontró Kant en un rasgo llamativo del discurso de Platón, a saber, en el hecho de que Platón maneja un «lenguaje elevado» (allí donde él habla sobre las ideas) «susceptible de

5 Op. cit., B 370.

6 Op. cit., B 371.

7 Entre los nuevos intérpretes de Kant, Volker Gerhardt llama la atención sobre este punto: Immanuel Kant. Vernunft und Leben (Reclam, Stuttgart, 2002), p. 184. 
una interpretación más suave, de una interpretación acorde con la naturaleza de las cosas $\|^{8}$.

Sobre su comprensión práctica de la doctrina de las ideas de Platón, Kant ofrece dos confirmaciones convincentes al respecto. La primera pertenece a la filosofía moral y la segunda a la filosofía política.

Por lo que respecta a la primera, Kant se refiere a la cuestión de la virtud. El intento de resolver la cuestión de la virtud tomando como fundamento y punto de partida la experiencia, y derivar consecuentemente a partir de ella el concepto de virtud, está condenado al fracaso por el hecho de que por medio de este proceder empírico se convierte «la virtud en algo ambiguo y mudable según el tiempo y las circunstancias, en un sinsentido inservible para construir una regla». Según la interpretación de Kant, Platón señala aquí el camino correcto. Si se quiere alcanzar lo que en relación con la virtud es enteramente cierto, lo que es completamente indiscutido, entonces se tiene que aceptar la idea de la virtud, que es el único "original verdadero», aquello que todo hombre tiene a priori en su propia cabeza como una medida inalterable e indiscutible de la acción moral (o inmoral). Sólo ella es aquel modelo en virtud del cual es posible «todo juicio sobre el valor moral o lo inmoral» en general. Esta idea está «necesariamente en la base de todo acercamiento a la perfección moral» con independencia de que a este acercamiento se opongan numerosos impedimentos ocultos en la naturaleza humana. Pues «que un hombre jamás actúe de forma adecuada a aquello que la idea de la virtud contiene, no demuestra que este pensamiento sea quimérico»?.

A partir de esto se puede ver que Kant toma la doctrina de las ideas de Platón como el único punto de apoyo para liberar la cuestión de la virtud de todo lo empírico y para fortalecer la propia posición apriorística en relación con la virtud entendida como regla inequívoca y el criterio indiscutible para la acción moral. En esta medida no sería exagerado decir que Kant considera a Platón

\footnotetext{
${ }^{8}$ Immanuel Kant. Crítica de la razón pura, B 371, nota. En esta nota Kant manifiesta con claridad, que es completamente consciente del papel que juegan las ideas platónicas dentro de la filosofía teórica. Aparte de eso señala los puntos débiles de la doctrina de las ideas de Platón, que él ve en su «deducción mística» y en el hecho de que Platón las hipostasiara.

9 Op. cit., B 372.
} 
como un particular señalizador del camino hacia su propia Crítica de la razón práctica, hacia ese desplazamiento incomparable y sin parangón del problema moral a un terreno completamente nuevo ${ }^{10}$.

La segunda confirmación de la comprensión filosófico-práctica kantiana de la doctrina de las ideas de Platón pertenece, como ya hemos señalado anteriormente, a la filosofía política y remite por supuesto al pensamiento platónico del Estado ideal. A este respecto, la relación de Kant con Platón es doble. Por un lado, Kant se opone al irreflexivo e imprudente poco aprecio de la idea platónica del Estado ideal que sobre todo localiza en Brucker ${ }^{11}$, quien consideraba irrisoria la afirmación de Platón de que el príncipe nunca gobernará bien si no participa de las ideas. Semejante burla sobre una de las ideas políticas centrales de Platón es desactivada por Kant con el siguiente consejo: «en vez de dejar a un lado como inútil este pensamiento con el mísero y perjudicial pretexto de ser impracticable, sería mejor tenerlo más en cuenta e iluminarlo (allí donde el gran filósofo nos deja desamparados) con nuevos esfuerzos» ${ }^{12}$.

Pero a Kant no le es suficiente proteger a Platón de las objeciones superficiales o incluso de las burlas públicas ${ }^{13}$. Él da aún un paso más, y con ello se muestra el otro lado de su comprensión de Platón como pensador político. Su defensa de Platón le sirvió como ocasión para exponer precisamente en ese lugar y gracias a esa oportunidad la idea central de su propia filosofía política. De este modo expresaba de una manera indirecta pero potente su reconocimiento grande y especial a Platón, por cuanto que Kant aprueba completamente el método del idealismo político platónico, aunque no en lo que respecta a su contenido, ya que era consciente de que él como filósofo político se movía en circunstancias históricas completamente distintas. Ningún intérprete del pensamiento polí-

${ }^{10}$ Véase el tercer apartado del libro anteriormente citado de Wichmann, en especial las páginas 77-79 donde se trata la libertad moral y la incondicionalidad moral.

${ }^{11}$ En toda su amplia obra Kant sólo menciona este nombre una única vez y precisamente en este pasaje. La ironía quiso que de ese modo Kant ayudara a que Brucker no cayera por completo en el olvido.

12 Immanuel Kant. Crítica de la razón pura, B 372.

13 Tales críticas se realizan aún hoy en día en nombre de la empiría. Kant ha destruido para siempre estas objeciones cuando decía, defendiendo al mismo tiempo el oficio del filósofo: «Pues nada hay más pernicioso y más indigno de un filósofo que la plebeya apelación a una presunta experiencia contraria...» (Op. cit., B 373). 
tico de Kant puede poner en duda que el siguiente juicio, que formula Kant en la Crítica de la razón pura sobre Platón, representa el núcleo de toda su filosofía política, la que desarrollará después en sus artículos y tratados de la década de los años 80 y 90 del siglo XVIII, así como en su Metafisica de las costumbres de 1797 de manera extensa: «una constitución que promueva la mayor libertad humana de acuerdo con leyes que hagan que la libertad de cada uno sea compatible con la de los demás (no una constitución que promueva la mayor felicidad, pues ésta se seguirá por sí sola) es, como mínimo, una idea necesaria que ha de servir de base no sólo al primer esbozo de una constitución política, sino también a todas las leyes. En ello debe hacerse inicialmente abstracción de los obstáculos actuales, que acaso no surjan inevitablemente de la naturaleza humana, sino más bien por no haber atendido a las ideas auténticas en la legislación» ${ }^{14}$.

De manera análoga a lo que había dicho sobre la idea de la virtud, a saber, que ella es un fundamento indispensable para toda aproximación a la perfección moral, Kant considera ahora también que la idea de la constitución civil que descansa sobre la libertad mayor posible es el modelo con arreglo al cual se puede realizar un acercamiento progresivo a la perfección política tanto como sea posible. Kant sostiene firmemente la idea de que incluso los castigos y las penas serían cada vez menos numerosos si la legislación y el gobierno estuvieran más en consonancia con dicha idea, de modo que también comparte a su manera y de una forma más suave la convicción de Platón que él interpreta del siguiente modo: «cuanto más de acuerdo con esta idea estuvieran organizados la legislación y el gobierno, tanto más raros serían los castigos» ${ }^{15}$. Y cuando se trata del abismo infranqueable entre la idea política y su realización, Kant hace notar que la profundidad de ese abismo no se puede o no se debe calibrar de antemano, ofreciendo en ello un argumento que no se puede calificar sino de magnífico: la libertad es aquello que es capaz de franquear todo límite dado ${ }^{16}$.

La idea kantiana de un Estado que descansa sobre la libertad humana, la mayor posible, regulada por medio de leyes, presenta realmente, expresado con la ayuda de la nueva terminología de Kant, la idea de un Estado de derecho en el que se garantiza efectividad a los inviolables e invulnerables derechos huma-

14 Op. cit., B 373.

15 Ibid.

16 Véase Op. cit., B 374. 
nos. Por eso no es extraño ni carente de fundamento que Kant sea alineado entre aquellos filósofos políticos y filósofos del derecho que defendieron inquebrantablemente y justificaron constantemente el Estado de derecho como la idea y como el bien político jurídico supremo.

Aunque Kant haya interpretado la doctrina de las ideas de Platón, en consonancia con su propio interés filosófico, fundamentalmente desde la perspectiva de la filosofía práctica, no olvidó con ello que Platón habló sobre las ideas también en relación con la naturaleza y en relación con su origen y creaciones ${ }^{17}$. Pero no es necesario que nos ocupemos ahora detenidamente de esta cuestión, a pesar de que también esto completa la imagen kantiana de Platón en su primera Crítica. Sin embargo es necesario mencionar, por último, dos puntos para la completar esta imagen.

El primer punto se refiere al modo como Kant resalta el impulso espiritual de Platón gracias al cual asciende «desde la consideración del cosmos físico como copia hasta su conexión arquitectónica según fines, es decir, según ideas» ${ }^{18}$. En este fuerte impulso del pensar vio Kant un mérito especial de Platón, por cuanto que invita al mismo tiempo a honorar y seguir estos esfuerzos.

El segundo punto merece una mayor atención por nuestra parte, porque deja aparecer la imagen que tiene Kant de Platón bajo una perspectiva especial. En conexión con su exposición de las ideas, que fue inspirada y motivada por Platón, y que fue llevada a cabo resaltando lo práctico, resaltando aquella especie de causalidad que él designa como causalidad por libertad, Kant considera adecuado e imprescindible unir a esta exposición la peculiar dignidad de la filosofía. Esta dignidad, que presupone la mirada dirigida al mundo de las ideas (para lo cual Kant advierte: sin deducción ni hipostatización místicas), protege la filosofía para que no se sumerja en la mera empiria carente de pensamiento y poseída por un puro pragmatismo. Apoyándose en Platón, sin que ello signifique empero una plena identificación con el creador de la doctrina de las ideas, que sería por lo demás incompatible con la intención crítica de su filosofía, Kant fue el verdadero defensor de la mencionada dignidad de la filosofía. Permaneció fiel a esta idea, cuando al final de sus reflexiones sobre Platón y como último rasgo de su ima-

17 Véase Ibid.

18 Op. cit., B 375. 
gen de Platón, declaró en la Crítica de la razón pura que «es muy reprochable tomar las leyes relativas a lo que se debe hacer de aquello que se hace o bien limitarlas en virtud de esto último» ${ }^{19}$.

Pues bien, si nosotros prestamos atención a todo lo dicho hasta aquí, no podemos evitar la impresión de que Kant, cuando elaboró su imagen de Platón, ofreció al mismo tiempo algunos de los rasgos fundamentales de su propio retrato en cuanto filósofo crítico. Esto fue ciertamente posible porque tanto él como Platón mantienen un profundo parentesco intelectual, del que el propio Kant era plenamente consciente. En este sentido no se equivocaba Wichmann cuando concluía su estudio comparativo sobre Platón y Kant diciendo que ambos autores se complementan mutuamente de tal modo que para poder comprender a Platón antes hay que pasar por la escuela del pensamiento kantiano, y para poder vivenciar a Kant antes se ha de morar en el espíritu de Platón.

\section{Bibliografía}

KanT, I. (1968). Kritik der reinen Vernunft, Kant: Werke in zwoelf Baenden; Baende IIIIV. Herausgegeben von Wilhelm Weischedel, Frankfurt am Main: Suhrkamp Verlag,

GerhaRDT. V. (2002). Immanuel Kant. Vernunft und Leben, Stuttgart: Reclam.

NATORP, P. (1994). Platos Ideenlehre, Hamburg : Felix Meiner.

Wichmann, O. (1920). Platon und Kant. Eine vergleichende Studie, Berlin: Weidmannsche Buchhandlung.

Recibido: $14 / 04 / 2010$

Aceptado: 12/06/2010

19 Ibid. 